\title{
Robust optimal stabilization of the Brockett integrator via a hybrid feedback
}

\author{
C. Prieur $^{1}$, E. Trélat ${ }^{2}$ \\ 1 LAAS-CNRS, 7 avenue du Colonel Roche, 31077 Toulouse, France, Tel: (+33) \\ 5613364 80, Fax: (+33) 561336969 e-mail: christophe.prieur@laas.fr \\ ${ }^{2}$ Univ. Paris-Sud, Labo. AN-EDP, Bat. 425, 91405 Orsay Cedex, France, Tel: \\ (+33) 1691566 73, Fax: (+33) 169156718 e-mail: emmanuel.trelat@math.u-psud.fr
}

Received: date / Revised version: date

\begin{abstract}
The problem of semi-global minimal time robust stabilization of the Brockett integrator (also called Heisenberg system) is addressed and solved by means of a hybrid state feedback law. It is shown that the solutions of the closed-loop system converge to the origin, in quasi-minimal time (for a given bound on the controller), with a robustness property with respect to small measurement noises, external disturbances and actuator noises.
\end{abstract}

Key words Brockett integrator - optimal control - hybrid feedback robust stabilization - measurement errors - actuator noise - external disturbances 


\section{Introduction}

Consider the so-called Brockett system in $\mathbb{R}^{3}$

$$
\dot{x}=u_{1} f_{1}(x)+u_{2} f_{2}(x)
$$

where, denoting $x=\left(x_{1}, x_{2}, x_{3}\right)$,

$$
f_{1}=\frac{\partial}{\partial x_{1}}+x_{2} \frac{\partial}{\partial x_{3}}, f_{2}=\frac{\partial}{\partial x_{2}}-x_{1} \frac{\partial}{\partial x_{3}}
$$

and the control function $u=\left(u_{1}, u_{2}\right)$ satisfies the constraint

$$
u_{1}^{2}+u_{2}^{2} \leq 1
$$

The system (1), together with the constraint (3), is said to be globally asymptotically stabilizable at the origin, if, for each point $x$ of $\mathbb{R}^{3}$, there exists a control law $x \mapsto u(x)$, satisfying the constraint (3), such that the solution of (1), associated to this control law, and starting from $x$, tends to 0 as $t$ tends to $+\infty$.

Note that, due to Brockett's condition (see [9, Theorem 1, (iii)]), there does not exist any continuous stabilizing feedback law $x \mapsto u(x)$ for (1). Several control laws have been however derived for control systems of this type (see e.g. $[16,13,3]$ and references therein).

The robust asymptotic stabilization problem is under current and active research. There exists a large variety of control laws that solve the robust asymptotic stabilization problem, such as discontinuous sampling feedbacks $[10,25]$, time varying control laws $[12,11,17,18]$, patchy feedbacks (as in [1]), 
SRS feedbacks [24], ..., yielding different robustness properties depending on the errors under consideration in these papers.

The class of feedbacks considered here consists of feedback laws mixing discrete and continuous components. It gives rise to closed-loop systems with a hybrid term, studied e.g. in $[28,6]$. The use of such a class of feedbacks for the stabilization of nonlinear systems (a priori without discrete state) first appeared in [20]. It allows to design a switching strategy between different smooth control laws defined on a partition of the state space. This idea of defining the control smoothly part by part, and switching between the different components, is usual in nonlinear control theory (see e.g. $[27,2]$ ). It has also been applied in [23] to chained systems with two commands. However, in this paper, we provide, as in [23], a hybrid feedback law for the system (1) with the constraint (3), that is not only robustly stabilizing, but also enjoys a minimal time property. It consists, on the one hand, of a minimal time controller, that is smooth on a part of the state space, and on the other, of a second controller, defined on the complement of this part, and we define the switching strategy between both control laws. Our strategy enjoys a minimal time property, and robustness with respect to (small) measurement noises, actuator errors and external disturbances.

To this aim, we first solve the minimal time control problem, for the system (1) with the constraint (3), and then, we define a hybrid feedback law, using a suitable switching strategy (namely, a hysteresis) between this 
minimal time controller and another controller defined on a neighborhood of the singular set of the optimal control law.

The paper is organized as follows. First, we recall a notion of solution adapted to hybrid feedback laws, and make precise the notion of stabilization via a minimal time hybrid feedback law. We then state the main result, namely, that there exists a minimal time hybrid feedback law stabilizing semi-globally and robustly the origin for the system (1) with the constraint (3) (see Section 3). The rest of the paper is devoted to the proof of this result. We recall how to solve explicitly the minimal time problem for the system (1) with the constraint (3), and define our "local" minimal time control law in Section 4.1, and the "global" one in Section 4.2. The hybrid feedback law is defined in Section 5, and the main result is proved in Section 6.

\section{Class of controllers and notion of solution}

Let $n$ and $m$ be positive integers, and $f: \mathbb{R}^{n} \times \mathbb{R}^{m} \rightarrow \mathbb{R}^{n}$ be a continuous function, which is locally Lipschitzian in $x \in \mathbb{R}^{n}$, uniformly for $u \in \mathbb{R}^{m}$, such that $f(0,0)=0$. Consider the control system

$$
\dot{x}=f(x, u) \text {. }
$$

The Brockett system (1) investigated in this paper is of the form (4).

The hybrid feedback laws under consideration admit the following description (see $[28,6])$

$$
u=k\left(x, s_{d}\right), s_{d}=k_{d}\left(x, s_{d}^{-}\right)
$$


where $s_{d}$ evolves in the finite set $\{1,2\}$, the function $k: \mathbb{R}^{n} \times\{1,2\} \rightarrow \mathbb{R}^{m}$ is a continuous function of $x$, for each fixed $s_{d}$, and $k_{d}: \mathbb{R}^{n} \times\{1,2\} \rightarrow\{1,2\}$ is a function. The notation $s_{d}^{-}$stands, at this stage only formally, for

$$
s_{d}^{-}(t)=\lim _{s<t} s_{d}(s)
$$

The set $\{1,2\}$ is endowed with the discrete topology, i.e. every set is an open set. The above controller is hybrid, due to the presence of the discrete dynamics of $s_{d}$. It gives rise to a non-classical ordinary differential equation describing the dynamics of the closed-loop system.

We next recall the notion of robustness to a small noise (see [26]). Let $L_{l o c}^{\infty}\left(\mathbb{R}^{n} \times[0,+\infty) ; \mathbb{R}^{n}\right)$ denote the set of essentially locally bounded functions, with values in $\mathbb{R}^{n}$.

Consider two functions $e$ and $d$ satisfying the following regularity assumptions:

$$
\begin{aligned}
& e(\cdot, \cdot), d(\cdot, \cdot) \in L_{l o c}^{\infty}\left(\mathbb{R}^{n} \times[0,+\infty) ; \mathbb{R}^{n}\right), \\
& e(\cdot, t), d(\cdot, t) \in C^{0}\left(\mathbb{R}^{n}, \mathbb{R}^{n}\right), \quad \forall t \in[0,+\infty) .
\end{aligned}
$$

We introduce these functions as a measurement noise $e$ and an external disturbance $d$, and define the perturbed system ${ }^{1}$ with $u$ given by (5), by

$$
\begin{aligned}
\dot{x}(t) & =f\left(x(t), k\left(x(t)+e(x(t), t), s_{d}(t)\right)\right)+d(x(t), t), \\
s_{d}(t) & =k_{d}\left(x(t)+e(x(t), t), s_{d}^{-}(t)\right) .
\end{aligned}
$$

The notion of a solution of such a hybrid perturbed system has been wellstudied in the literature (see e.g. $[6,7,15,28,22,23]$ ). To be self-contained,

\footnotetext{
${ }^{1}$ we can also consider an actuator noise (see e.g. $[14,20]$ ).
} 
we recall the definition of a solution of (8) and we introduce a non-empty set $\mathcal{R C}$ strictly contained in $\mathbb{R}^{n} \times\{1,2\}$.

Definition 1 Given $T>0$ and $\left(x_{0}, s_{0}\right) \in \mathbb{R}^{n} \times\{1,2\}$, we say that $\left(x, s_{d}\right)$ is a solution of (8) on $[0, T)$, starting from $\left(x_{0}, s_{0}\right)$, if the following conditions hold:

- the map $x$ is absolutely continuous on $[0, T)$;

- there holds, for almost every $t \in[0, T)$,

$$
\dot{x}(t)=f\left(x(t), k\left(x(t)+e(x(t), t), s_{d}(t)\right)\right)+d(x(t), t) ;
$$

- for every $t \in[0, T)$ such that $\left(x(t), s_{d}(t)\right) \in \mathcal{R C}$, the map $s_{d}$ is rightcontinuous at $t$;

- for every $t \in(0, T)$ such that $s_{d}^{-}(t)$ exists, one has

$$
s_{d}(t)=k_{d}\left(x(t)+e(x(t), t), s_{d}^{-}(t)\right)
$$

- there holds $x(0)=x_{0}$, and $s_{d}(0)=k_{d}\left(x_{0}+e\left(x_{0}, 0\right), s_{0}\right)$.

We next define the concept of stabilization of (4) by a minimal time hybrid feedback law sharing a robustness property with respect to measurement noises and external disturbances. The usual Euclidean norm in $\mathbb{R}^{n}$ is denoted by $|\cdot|$. Recall that a function of class $\mathcal{K}_{\infty}$ is a function $\delta$ : $[0,+\infty) \rightarrow[0,+\infty)$ which is continuous, increasing, satisfying $\delta(0)=0$ and $\lim _{R \rightarrow+\infty} \delta(R)=+\infty$.

Definition 2 Let $\rho: \mathbb{R}^{n} \rightarrow \mathbb{R}$ be a continuous function, which is positive outside 0 . We say that the completeness assumption holds for $\rho$ if, for all 
$(e, d)$ satisfying the regularity assumptions (7) and

$$
\sup _{[0,+\infty)}|e(x, \cdot)| \leq \rho(x), \operatorname{esssup}_{[0,+\infty)}|d(x, \cdot)| \leq \rho(x), \forall x \in \mathbb{R}^{n},
$$

for all $\left(x_{0}, s_{0}\right) \in \mathbb{R}^{n} \times\{1,2\}$, there exists a maximal solution of (8) on $[0,+\infty)$, starting from $\left(x_{0}, s_{0}\right)$.

Definition 3 We say that the uniform finite time convergence property holds if there exists a continuous function $\rho: \mathbb{R}^{n} \rightarrow \mathbb{R}$, positive outside 0 , satisfying the completeness assumption, and if there exists a function $\delta$ of class $\mathcal{K}_{\infty}$, so that, for every $R>0$, there exists $\tau>0$ such that, for all functions e,d satisfying the regularity assumptions (7) and inequalities (10) for this function $\rho$, for every $x_{0} \in \mathbb{R}^{n},\left|x_{0}\right| \leq R$, and every $s_{0} \in\{1,2\}$, the maximal solution $\left(x, s_{d}\right)$ of $(8)$, starting from $\left(x_{0}, s_{0}\right)$, satisfies

$$
|x(t)| \leq \delta(R), \forall t \geq 0
$$

and

$$
x(t)=0, \forall t \geq \tau
$$

Definition 4 The origin is said to be a semi-globally minimal time robustly stabilizable equilibrium for the system (4) if, for every $\varepsilon>0$ and every compact subset $K \subset \mathbb{R}^{n}$, there exists a hybrid feedback law $\left(u, k_{d}\right): \mathbb{R}^{n} \times$ $\{1,2\} \rightarrow \mathbb{R}^{m} \times\{1,2\}$ satisfying the constraint

$$
\left\|k\left(x, s_{d}\right)\right\| \leq 1
$$

where $\|\cdot\|$ stands for the Euclidian norm in $\mathbb{R}^{m}$, such that:

- the uniform finite time convergence property holds; 
- there exists a continuous function $\rho_{\varepsilon, K}: \mathbb{R}^{n} \rightarrow \mathbb{R}$, positive outside 0 , such that, for all functions e, $d$ satisfying the regularity assumptions (7) and inequalities (10) for $\rho=\rho_{\varepsilon, K}$, for every $x_{0} \in K$, the maximal solution of (8), starting from $x_{0}$, reaches the origin within time $T\left(x_{0}\right)+\varepsilon$, where $T\left(x_{0}\right)$ denotes the minimal time to steer the system (4) from $x_{0}$ to the origin, with the constraint $\|u\| \leq 1$.

\section{Main result}

Theorem 1 The origin is a semi-globally minimal time robustly stabilizable equilibrium for the system (1) with the constraint (3).

Remark 1 Some observations are in order.

1. An explicit expression for the hybrid feedback law $\left(u, k_{d}\right)$ is given in Section 5 .

2. Note that the problem of global robust minimal time stabilization (i.e. $K=\mathbb{R}^{3}$ in Definition 4) cannot be achieved because measurement noises may then accumulate and slow down the solution reaching the origin (compare with [8]). However, we provide a globally asymptotically stabilizing hybrid controller, in the sense that the constant $R>0$ of the Definition 3 can be arbitrarily large.

3. Note that the conclusion of Theorem 1 does not hold if, in (10), the supremum sup is relaxed by esssup (see [20, Theorem 4.2], where it is proved, in an analogous situation, that there exists a measurement noise 
$e$ satisfying esssup $|e|=0$, sup $|e| \neq 0$, such that the origin of the perturbed closed-loop system is not an attractive equilibrium).

Intuitively, the strategy is as follows. For $x \in \mathbb{R}^{3}$, let $T(x)$ denote the minimal time needed to steer the system (1) from $x$ to the origin, with the constraint (3). The corresponding minimal time feedback controller, called local controller, happens to be continuous (even analytic) on $\mathbb{R}^{n} \backslash\left\{x_{1}=\right.$ $\left.x_{2}=0\right\}$. It is therefore necessary to use another controller, called global controller, in a neigborhood $\Omega$ of the line $\left\{x_{1}=x_{2}=0\right\}$. More precisely, $\Omega$ will be constructed so as to be cylindric around this line, and conic near the origin (see Fig. 4 further). In this neighborhood, we define an adequate switching strategy. Notice that $\Omega$ is arbitrarily thin, and thus the time $\varepsilon$ needed for the traversing of $\Omega$ is arbitrarily small, uniformly with respect to the initial condition. Therefore, starting from an initial point $x$, the time needed to join the origin, using this hybrid strategy, is equal to $T(x)+\varepsilon$.

The rest of the paper is organized as follows. We define the local controller in Section 4.1 and the global one in Section 4.2. The switching strategy between these feedback laws by means of a hysteresis is explained in Section 5. Theorem 1 is proved in Section 6 . 


\section{The components of the hysteresis}

\subsection{The local controller}

In this section, we define and compute the local controller, and give some properties of the Carathéodory solutions of (1) with this feedback law.

Consider the Brockett system (1). It is a standard fact that the minimal time problem for the system (1), with the constraint (3), is equivalent to the sub-Riemannian problem in $\mathbb{R}^{3}$ associated to the vector fields $f_{1}$ and $f_{2}$ (see for instance [5]), and moreover, the minimal time $T(x)$ needed to steer the origin to a point $x \in \mathbb{R}^{3}$ is equal to the sub-Riemannian distance of $x$ to the origin. Using this fact, the function $T$ may be computed explicitly, and we recall the following result of [4].

Proposition 1 Consider the minimal time problem for the system (1) with the constraint (3). The minimal time $T(x)$ needed to steer a point $x=$ $\left(x_{1}, x_{2}, x_{3}\right) \in \mathbb{R}^{3}$ to the origin is given by

$$
T\left(x_{1}, x_{2}, x_{3}\right)=\frac{\theta}{\sqrt{\theta+\sin ^{2} \theta-\sin \theta \cos \theta}} \sqrt{x_{1}^{2}+x_{2}^{2}+2\left|x_{3}\right|},
$$

where $\theta=\theta\left(x_{1}, x_{2}, x_{3}\right)$ is the unique solution in $[0, \pi)$ of

$$
\frac{\theta-\sin \theta \cos \theta}{\sin ^{2} \theta}\left(x_{1}^{2}+x_{2}^{2}\right)=2\left|x_{3}\right| .
$$

Moreover, the function $T$ is continuous on $\mathbb{R}^{3}$, and is analytic outside the line $x_{1}=x_{2}=0$.

Remark 2 The function $g$, defined by

$$
g(\theta)=\frac{\theta-\sin \theta \cos \theta}{\sin ^{2} \theta}
$$


for $\theta \in[0, \pi)$, with the agreement that $g(0)=0$, is a monotone increasing diffeomorphism of $(0, \pi)$ onto $(0,+\infty)$. For every $\theta \in[0, \pi)$, define

$$
h(\theta)=\frac{\theta}{\sqrt{\theta+\sin ^{2} \theta-\sin \theta \cos \theta}},
$$

with the agreement that $h(0)=1$ (see Fig. 1).
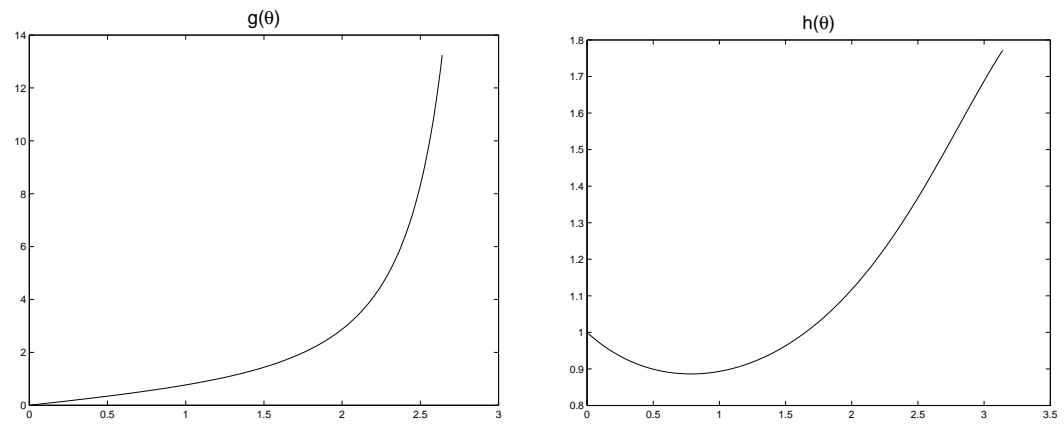

Fig. 1 The functions $g$ and $h$.

For every $s \in \mathbb{R}$, set $F(s)=h \circ g^{-1}(s)$. Then, there holds, outside the line $x_{1}=x_{2}=0$,

$$
T\left(x_{1}, x_{2}, x_{3}\right)=F\left(\frac{2\left|x_{3}\right|}{x_{1}^{2}+x_{2}^{2}}\right) \sqrt{x_{1}^{2}+x_{2}^{2}+2\left|x_{3}\right|} .
$$

It can be checked that $T$ is indeed analytic outside the line $x_{1}=x_{2}=0$, even though it is not immediate from this expression (see [4]).

Remark 3 Along the line $x_{1}=x_{2}=0$, there holds

$$
T\left(0,0, x_{3}\right)=\sqrt{2 \pi\left|x_{3}\right|}
$$

The singular set of the function $T$, i.e. the set where $T$ is not $C^{1}$, is the line $x_{1}=x_{2}=0$ in $\mathbb{R}^{3}$. More precisely, the gradients $\partial T / \partial x_{i}, i=1,2$, 
are discontinuous at every point $\left(0,0, x_{3}\right), x_{3} \neq 0$. In the context of subRiemannian geometry, this singular line can be interpreted as a cut-locus.

Actually, for every $x_{3} \neq 0$, there exists an infinite number of optimal trajectories steering the origin to the point $\left(0,0, x_{3}\right)$, given by the set of curves

$$
\begin{aligned}
& x_{1}(t)=\sqrt{\frac{\left|x_{3}\right|}{2 \pi}}(\sin (2 \pi t+\varphi)-\sin \varphi), \\
& x_{2}(t)=\sqrt{\frac{\left|x_{3}\right|}{2 \pi}}(\cos (2 \pi t+\varphi)-\cos \varphi), \\
& x_{3}(t)=x_{3} t-\frac{\left|x_{3}\right|}{2 \pi} \sin (2 \pi t),
\end{aligned}
$$

for $t \in[0,1]$ and $\varphi \in[0,2 \pi]$ (see for instance [4]).

Remark 4 Using the previous notations, there holds, for every $x=\left(x_{1}, x_{2}, x_{3}\right) \in$ $\mathbb{R}^{3}$,

$$
\begin{aligned}
T\left(x_{1}, x_{2}, x_{3}\right) & =h(\theta) \sqrt{x_{1}^{2}+x_{2}^{2}+\left|x_{3}\right|} \\
& =\left(\theta \frac{\cos \theta}{\sin \theta}\left(x_{1}^{2}+x_{2}^{2}\right)+2 \theta\left|x_{3}\right|\right)^{\frac{1}{2}}, \\
& =\frac{\theta}{\sin \theta} \sqrt{x_{1}^{2}+x_{2}^{2}}
\end{aligned}
$$

where $\theta=\theta\left(x_{1}, x_{2}, x_{3}\right)$ is the unique solution in $[0, \pi)$ of $(15)$.

A level set $\left\{\left(x_{1}, x_{2}, x_{3}\right) \in \mathbb{R}^{3} \mid T\left(x_{1}, x_{2}, x_{3}\right)=r\right\}$, where $r>0$, is drawn on Fig. 2. In the context of sub-Riemannian geometry, it represents the subRiemannian sphere centered at the origin, with radius $r$, in the Heisenberg case (see [5]). Observe that it is axial symmetric, with respect to the axis $\left(0 x_{3}\right)$.

On Fig. 3 are drawn intersections of different level sets of $T$ with a plane containing the axis $\left(0 x_{3}\right)$. 


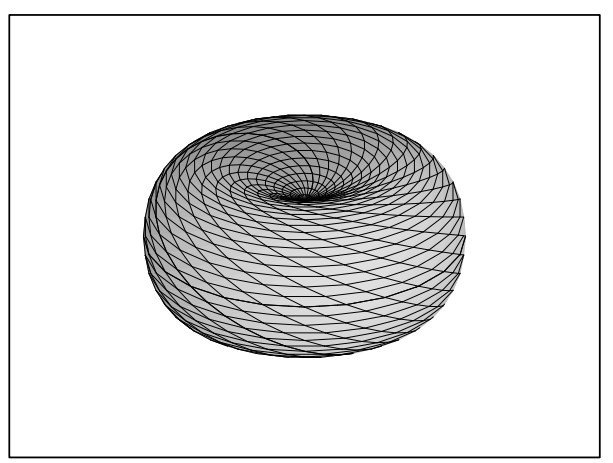

Fig. 2 Level set $\left\{\left(x_{1}, x_{2}, x_{3}\right) \in \mathbb{R}^{3} \mid T\left(x_{1}, x_{2}, x_{3}\right)=r\right\}$.

Remark 5 There exists a one-parameter group of dilations

$$
\delta_{\lambda}:\left(x_{1}, x_{2}, x_{3}\right) \mapsto\left(\lambda x_{1}, \lambda x_{2}, \lambda^{2} x_{3}\right)
$$

so that

$$
T\left(\delta_{\lambda} x\right)=|\lambda| T(x)
$$

for every $x \in \mathbb{R}^{3}$ (see [5]). This property can be observed on Fig. 3 .

It follows from the Pontryagin maximum principle and the HamiltonJacobi theory (see [19]) that the minimal time control functions, steering a point $x=\left(x_{1}, x_{2}, x_{3}\right) \in \mathbb{R}^{3}$ to the origin, are given by the closed-loop formula

$$
\begin{aligned}
& u_{1}(x)=-\frac{1}{2}\left\langle\nabla T(x), f_{1}(x)\right\rangle=-\frac{1}{2}\left(\frac{\partial T}{\partial x_{1}}+x_{2} \frac{\partial T}{\partial x_{3}}\right) \\
& u_{2}(x)=-\frac{1}{2}\left\langle\nabla T(x), f_{2}(x)\right\rangle=-\frac{1}{2}\left(\frac{\partial T}{\partial x_{2}}-x_{1} \frac{\partial T}{\partial x_{3}}\right)
\end{aligned}
$$

provided $T$ is $C^{1}$ at $x$. In particular, this holds outside the line $x_{1}=x_{2}=0$. 


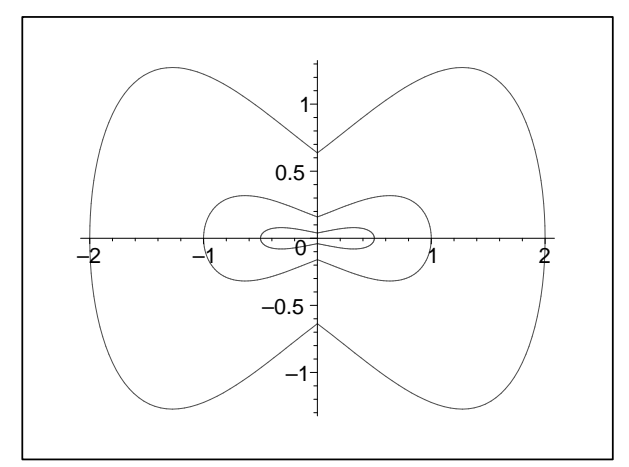

Fig. 3 Intersection of different level sets with a vertical plane.

Outside this line, the smoothness of this optimal controller ensures a robustness property of the stability. A switching strategy is then necessary between this optimal controller and another controller which has to be continuous in a neighborhood of the line $x_{1}=x_{2}=0$. This latter controller, denoted $u_{g}$, is called global controller, and is defined in Section 4.2, whereas the optimal one, denoted $u_{l}$, is called the local controller. The switching strategy is achieved by adding a dynamical discrete variable $s_{d}$ and using a hybrid feedback law (see Section 5).

Below, we give an explicit expression of the optimal controller (local controller), using the following lemma.

Lemma 1 For every $x=\left(x_{1}, x_{2}, x_{3}\right) \in \mathbb{R}^{3}$ such that $x_{1}^{2}+x_{2}^{2} \neq 0$, there holds

$$
\frac{\partial T}{\partial x_{i}}=\cos \theta \frac{x_{i}}{\sqrt{x_{1}^{2}+x_{2}^{2}}}, i=1,2, \quad \frac{\partial T}{\partial x_{3}}=\operatorname{sign}\left(x_{3}\right) \sin \theta
$$

where $\theta=\theta\left(x_{1}, x_{2}, x_{3}\right)$ is the unique solution in $[0, \pi)$ of $(15)$. 
Remark 6 Notice that, if $x_{1}^{2}+x_{2}^{2} \neq 0$, then $\theta\left(x_{1}, x_{2}, 0\right)=0$, and hence $\frac{\partial T}{\partial x_{3}}\left(x_{1}, x_{2}, 0\right)=0$.

Corollary 1 The minimal time controller $u_{l}=\left(u_{l 1}, u_{l 2}\right)$, steering a point $x=\left(x_{1}, x_{2}, x_{3}\right) \in \mathbb{R}^{3}$ such that $x_{1}^{2}+x_{2}^{2} \neq 0$ to the origin, writes $u_{l 1}(x)=-\frac{1}{2}\left(\frac{x_{1}}{\sqrt{x_{1}^{2}+x_{2}^{2}}} \cos \left(g^{-1}\left(\frac{2\left|x_{3}\right|}{x_{1}^{2}+x_{2}^{2}}\right)\right)+\operatorname{sign}\left(x_{3}\right) x_{2} \sin \left(g^{-1}\left(\frac{2\left|x_{3}\right|}{x_{1}^{2}+x_{2}^{2}}\right)\right)\right)$,
$u_{l 2}(x)=-\frac{1}{2}\left(\frac{x_{2}}{\sqrt{x_{1}^{2}+x_{2}^{2}}} \cos \left(g^{-1}\left(\frac{2\left|x_{3}\right|}{x_{1}^{2}+x_{2}^{2}}\right)\right)-\operatorname{sign}\left(x_{3}\right) x_{1} \sin \left(g^{-1}\left(\frac{2\left|x_{3}\right|}{x_{1}^{2}+x_{2}^{2}}\right)\right)\right)$,

where the function $g$ is defined by (16).

For all $M>0$ and $r>0$, we introduce the subset of $\mathbb{R}^{3}$

$$
\Omega_{M, r}=\left\{\left(x_{1}, x_{2}, x_{3}\right) \in \mathbb{R}^{3} \mid x_{1}^{2}+x_{2}^{2} \leq \min \left(r, M\left|x_{3}\right|\right)\right\}
$$

and denote its complement in $\mathbb{R}^{3}$ by $\Gamma_{M, r}$. Near the origin, $\Omega_{M, r}$ is a cone, otherwise it is a cylinder around the axis $\left(0 x_{3}\right)$ (see Fig. 4).

The following lemma follows immediately from the behavior of the optimal flow (see formulas (17) in Remark 3).

Lemma 2 There exist $M_{0}>0$ and $r_{0}>0$ such that, for all $M$ and $r$ satisfying $0<M<M_{0}$ and $0<r<r_{0}$, the subset $\Gamma_{M, r}$ is invariant by the feedback optimal controller $u_{l}$.

Now this local controller has been defined, we investigate the robustness properties of the system (1) in closed-loop with this controller. Given $e, d$ : $\mathbb{R}^{3} \times[0,+\infty) \rightarrow \mathbb{R}^{3}$, the perturbed closed-loop system under consideration 


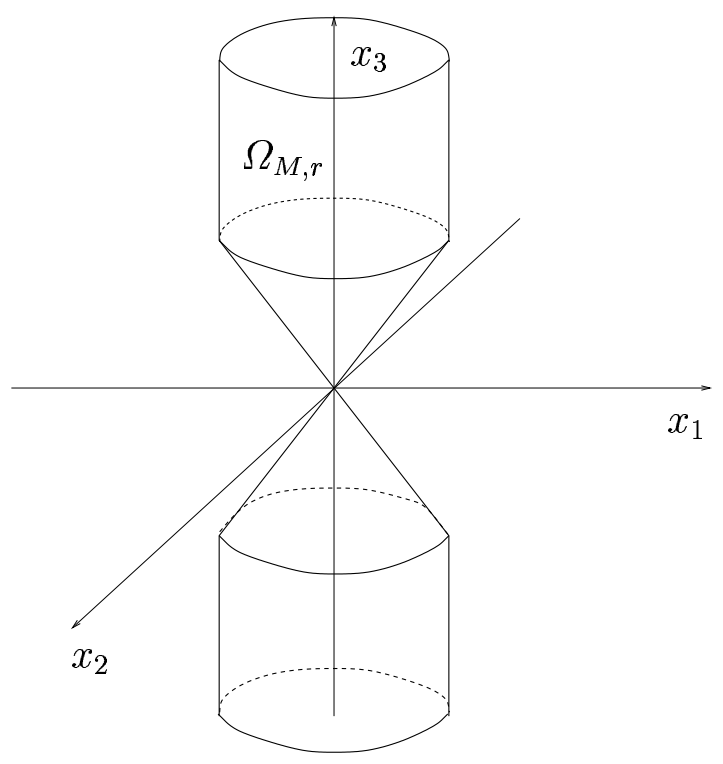

Fig. 4 Shape of $\Omega_{M, r}$.

in this section is of the form

$$
\dot{x}(t)=f\left(x(t), u_{l}(x(t)+e(x(t), t))\right)+d(x(t), t) .
$$

Below, a robust version of Lemma 2 is stated for every noise vanishing along the discontinuous set of the local controller. More precisely, all properties needed to state our main result are summarized in the following lemma.

Lemma 3 There exist a continuous function $\rho_{l}: \mathbb{R} \rightarrow \mathbb{R}$, positive outside 0 , and a continuous function $\delta_{l}:[0,+\infty) \rightarrow[0,+\infty)$ of class $\mathcal{K}_{\infty}$, such that:

- Stability: for all $M$ and $r$ satisfying $0<M<M_{0}$ and $0<r<r_{0}$, all $e, d: \mathbb{R}^{3} \times[0,+\infty) \rightarrow \mathbb{R}^{3}$ satisfying the regularity assumptions (7) and $\sup _{[0,+\infty)}|e(x, \cdot)| \leq \rho_{l}\left(x_{1}^{2}+x_{2}^{2}\right), \operatorname{esssup}_{[0,+\infty)}|d(x, \cdot)| \leq \rho_{l}\left(x_{1}^{2}+x_{2}^{2}\right), \forall x \in \mathbb{R}^{3}$ 
and for every $x_{0} \in \Gamma_{M, r}$, there exists a unique maximal Carathéodory solution $x(\cdot)$ of (24) on $[0,+\infty)$, starting from $x_{0}$, satisfying $x(t) \in \Gamma_{M, r}$, for every $t>0$;

- Finite-time convergence: for every $R>0$, there exists $\tau_{l}>0$ such that, for all e, $d: \mathbb{R}^{3} \times[0,+\infty) \rightarrow \mathbb{R}^{3}$ satisfying the regularity assumptions (7) and (25), for every $x_{0}$ in $\mathbb{R}^{3}$ with $\left|x_{0}\right| \leq R$, and every maximal solution $x(\cdot)$ of (24) starting from $x_{0}$, one has

$$
\begin{gathered}
|x(t)| \leq \delta_{l}(R), \forall t \geq 0, \\
x(t)=0, \forall t \geq \tau_{l},
\end{gathered}
$$

and

$$
\left\|u_{l}(x(t))\right\| \leq 1, \forall t \geq 0
$$

- Optimality: for every $\varepsilon>0$, and every compact subset $K \subset \mathbb{R}^{3}$, there exists a continuous function $\rho_{\varepsilon, K}: \mathbb{R}^{3} \rightarrow \mathbb{R}$, positive outside 0 , such that, for all e, $d: \mathbb{R}^{3} \times[0,+\infty) \rightarrow \mathbb{R}^{3}$ satisfying the regularity assumptions (7) and

$$
\begin{aligned}
& \sup _{[0,+\infty)}|e(x, \cdot)| \leq \min \left(\rho_{l}\left(x_{1}^{2}+x_{2}^{2}\right), \rho_{\varepsilon, K}(x)\right), \forall x \in \mathbb{R}^{3}, \\
& \operatorname{esssup}_{[0,+\infty)}|d(x, \cdot)| \leq \min \left(\rho_{l}\left(x_{1}^{2}+x_{2}^{2}\right), \rho_{\varepsilon, K}(x)\right), \forall x \in \mathbb{R}^{3},
\end{aligned}
$$

and for every $x_{0} \in K \cap \Gamma_{M, r}$, the solution of (24), starting from $x_{0}$, reaches the origin within time $T\left(x_{0}\right)+\varepsilon$.

Proof Since Carathéodory conditions hold for the system (24), the existence of a unique forward Carathéodory solution of (24), for every initial condition, is ensured. Note that, since the controller $u_{l}$ is the minimal time control 
steering $x$ to the origin under the constraint (3), the inequality (28) holds. Since the local controller $u_{l}$ defined by (22) is continuous on $\mathbb{R}^{3} \backslash\left\{x_{1}=\right.$ $\left.x_{2}=0\right\}$, Lemma 2 implies the existence of $\rho_{l}:[0,+\infty) \rightarrow[0,+\infty)$. The last part of the result follows from (21) and from the continuity of solutions with respect to disturbances.

\subsection{The global controller}

In this section we define the second component of the hysteresis, called global controller and denoted $u_{g}$. Moreover we give some basic properties of the Carathéodory solutions of the closed-loop system (4) with such a control law $u_{g}$.

Let us consider the feedback law

$$
u_{g 1}(x)=1, u_{g 2}(x)=0 \text {. }
$$

The closed-loop system considered in this section is of the form

$$
\dot{x}(t)=f\left(x(t), u_{g}(x(t)+e(x(t), t))\right)+d(x(t), t) .
$$

The following result, whose proof is obvious using (30), states that the trajectories of the system (31) enter the region $\Gamma_{M, r}$ in finite time, while remaining bounded up to this time.

Lemma 4 With the notations of Lemma 3, there exists a continuous function $\rho_{g}: \mathbb{R}^{3} \rightarrow \mathbb{R}$ satisfying

$$
\rho_{g}(x)>0, \forall x \neq 0
$$


such that, for every initial condition, the perturbed system (31), where e and $d$ are two arbitrary functions satisfying the regularity assumptions (7) and inequalities (10) with $\rho=\rho_{g}$, admits a unique Carathéodory solution, defined for all $t \geq 0$.

Moreover there exists a function $\delta_{g}$ of class $\mathcal{K}_{\infty}$ such that, for all $R>0$, and $M$ and $r$ satisfying $0<M<M_{0}$ and $0<r<r_{0}$, there exists a time $\tau_{g}=\tau_{g}(M, r, R)$ such that all Carathéodory solution $x$ of (31) starting from $x_{0}$, with $\left|x_{0}\right| \leq R$, satisfies

$$
\begin{gathered}
|x(t)| \leq \delta_{g}(R), \forall t \leq \tau_{g}, \\
x(t) \in \Gamma_{M, r}, \quad \forall t \geq \tau_{g},
\end{gathered}
$$

and

$$
\left\|u_{g}(x(t))\right\| \leq 1, \forall t \geq 0
$$

\section{Definition of the hybrid controller}

In this section we define the hybrid controller by using a hysteresis to connect both controllers defined in Sections 4.1 and 4.2 .

For every $i \in\{1, \ldots, 6\}$, let $M_{i}$ and $r_{i}$ so that

$$
\begin{gathered}
0<M_{6}<M_{5}<M_{4}<M_{3}<M_{2}<M_{1}<M_{0}, \\
0<r_{6}<r_{5}<r_{4}<r_{3}<r_{2}<r_{1}<r_{0} .
\end{gathered}
$$

For the sake of simplicity, in what follows we set $\Gamma_{i}=\Gamma_{M_{i}, r_{i}}$ and $\Omega_{i}=$ $\Omega_{M_{i}, r_{i}}$, for every $i \in\{1, \ldots, 6\}$. The hybrid controller $\left(k, k_{d}\right)$ is defined 
using the following hysteresis between $u_{l}$ and $u_{g}$ on $\Gamma_{5}$ and $\Gamma_{2}$ :

$$
\begin{aligned}
k: \mathbb{R}^{3} \times\{1,2\} & \rightarrow \mathbb{R}^{2} \\
\left(x, s_{d}\right) \mapsto & u_{l}(x) \text { if } s_{d}=1, \\
& u_{g}(x) \text { if } s_{d}=2,
\end{aligned}
$$

and

$$
\begin{aligned}
k_{d}: \mathbb{R}^{3} \times\{1,2\} \rightarrow & \{1,2\} \\
\left(x, s_{d}\right) \mapsto 1 \quad & \text { if } x \in \Gamma_{2}, \\
& s_{d} \quad \text { if } x \in \Gamma_{5} \backslash \Gamma_{2}, \\
2 & \text { if } x \notin \Gamma_{5} \cup\{0\} .
\end{aligned}
$$

\section{Properties of the solutions and proof of Theorem 1}

\subsection{Properties of the solutions}

In this section, we study some properties of the solutions of the perturbed system (8) in closed-loop with the hybrid controller (37) and (38). Let $\varepsilon>0$ and $K$ be a compact subset of $\mathbb{R}^{n}$. We set ${ }^{2}$

$$
\mathcal{R C}=\mathbb{R}^{3} \times\{1,2\} \backslash\left(\left(\operatorname{clos}\left(\Gamma_{3}\right) \backslash \Gamma_{1}\right) \times\{2\} \bigcup\left(\operatorname{clos}\left(\Gamma_{6}\right) \backslash \Gamma_{4}\right) \times\{1\}\right)
$$

(see [21] for a similar but simpler situation). Let $\rho: \mathbb{R}^{3} \rightarrow \mathbb{R}$ be a continuous function, positive outside 0 , so that:

- for every $x \in \Gamma_{6}$,

$$
\rho(x) \leq \min \left(\rho_{l}\left(x_{1}^{2}+x_{2}^{2}\right), \rho_{\varepsilon, K}(x)\right),
$$

\footnotetext{
${ }^{2}$ For a given subset $\Omega$ of $\mathbb{R}^{n}$, the closure, the interior and the boundary of $\Omega$ are denoted by $\operatorname{clos}(\Omega), \operatorname{int}(\Omega)$ and $\partial \Omega$, respectively.
} 
and, for every $x \in \mathbb{R}^{3} \backslash \Gamma_{1}$,

$$
\rho(x) \leq \rho_{g}(x)
$$

where $\rho_{l}, \rho_{\varepsilon, K}$ and $\rho_{g}$ are defined in Lemma 3 and Lemma 4;

- the following implication holds, for every $i \in\{1, \cdots, 5\}$, and every $e \in \mathbb{R}^{3}$ so that $|e| \leq \rho(x)$

$$
x+e \in \Gamma_{i} \Rightarrow x \in \Gamma_{i+1}
$$

- the following implication holds, for every $i \in\{2, \cdots, 6\}$, and every $e \in \mathbb{R}^{3}$ so that $|e| \leq \rho(x)$

$$
x+e \notin \Gamma_{i} \Rightarrow x \notin \Gamma_{i-1} .
$$

Let $e$ and $d$ be two functions satisfying the regularity assumptions (7) and (10). We now state, without proof, a series of four preliminary results, which are instrumental to prove Theorem 1 . They concern general properties of solutions of hybrid systems, and the arguments are conceptually analogous to those of $[21,23]$.

Lemma 5 For all $\left(x_{0}, s_{0}\right) \in \mathbb{R}^{3} \times\{1,2\}$, there exists a solution of (8), in closed-loop with (37) and (38), starting from $\left(x_{0}, s_{0}\right)$.

To describe further properties of the trajectories of the considered system, we recall the definition of a switch.

Definition 5 A map $s_{d}:[0, T) \rightarrow\{1,2\}$ is said to have a switch at time $t$ if $s_{d}$ is not continuous at $t$. 
Let $\left(x, s_{d}\right)$ be a solution of (8) in closed-loop with (37) and (38). To address the problem of locating the points where $s_{d}$ may have a switch, consider the sets

$$
\begin{aligned}
& s_{d}^{p}(t)=\left\{s \mid \exists t_{n} \in[t, T), t_{n} \underset{n \rightarrow \infty}{\longrightarrow} t, s_{d}\left(t_{n}\right) \underset{n \rightarrow \infty}{\longrightarrow} s\right\}, \\
& s_{d}^{m}(t)=\left\{s \mid \exists t_{n} \in\left[t_{0}, t\right], t_{n} \underset{n \rightarrow \infty}{\longrightarrow} t, s_{d}\left(t_{n}\right) \underset{n \rightarrow \infty}{\longrightarrow} s\right\},
\end{aligned}
$$

for every $t$ in $(0, T)$.

Lemma 6 Let $\left(x, s_{d}\right)$ be a solution of (8), in closed-loop with (37) and (38), such that $s_{d}$ has a switch at time $t \in(0, T)$.

- If the switch is such that $2 \in s_{d}^{m}(t)$ and $1 \in s_{d}^{p}(t)$, then $x(t) \in \cos \left(\Gamma_{3}\right) \backslash$ $\Gamma_{1}$

- If the switch is such that $1 \in s_{d}^{m}(t)$ and $2 \in s_{d}^{p}(t)$, then $x(t) \in \cos \left(\Gamma_{6}\right) \backslash$ $\Gamma_{4}$.

Lemma 7 Let $\left(x, s_{d}\right)$ be a maximal solution of (8), in closed-loop with (37) and (38), defined on $[0, T)$. If $T<+\infty$, then $\lim \sup _{t \rightarrow T}|x(t)|=+\infty$.

Lemma 8 Let $\left(x, s_{d}\right)$ be a maximal solution of (8), in closed-loop with (37) and (38), defined on $[0, T)$. Then $T=+\infty$, and only the following cases may occur:

1. there exists no switch, and $x$ is a Carathéodory solution of (24) on $[0,+\infty)$, contained in $\Gamma_{6}$;

2. there exists a time $\sigma$ in $(0,+\infty)$ such that

- $x$ is a Carathéodory solution of (31) on $[0, \sigma)$, and is not in $\Gamma_{1}$;

- $x$ is a Carathéodory solution of (24) on $[\sigma,+\infty)$, contained in $\Gamma_{6}$; 
3. there exist two switches $\sigma_{1}$ and $\sigma_{2}$ in $(0,+\infty)$ such that

- $x$ is a Carathéodory solution of (24) on $\left[0, \sigma_{1}\right)$, contained in $\Gamma_{6} \backslash \Gamma_{1}$;

- $x$ is a Carathéodory solution of (31) on $\left[\sigma_{1}, \sigma_{2}\right)$, and is not in $\Gamma_{1}$;

- $x$ is a solution of (24) on $\left[\sigma_{2},+\infty\right)$, contained in $\Gamma_{6}$.

Remark 7 As a consequence of Lemma 8, every solution of the perturbed system switches at most two times.

\subsection{Proof of Theorem 1}

Let $\varepsilon$ be a positive real number, and $K$ be a compact subset of $\mathbb{R}^{n}$.

Consider $M_{0}>0$ and $r_{0}>0$ given by Lemma $2, M_{i}, r_{i}$ for every

$i \in\{1, \ldots, 6\}$ satisfying (36), and $\rho: \mathbb{R}^{3} \rightarrow \mathbb{R}$ satisfying (40)-(43). Up to reducing $M_{0}$ and $r_{0}$, we assume that

every solution of (31) starting from $\Omega_{6}$ enters $\Gamma_{0}$ in time less than $\varepsilon$,

and

every solution of (24) starting from $\Gamma_{6}$ enters $\Gamma_{1}$ in time less than $\varepsilon$. (47)

The proof splits into three parts.

\section{Completeness}

Existence of solutions follows from Lemma 5, and maximality from Lemma

8. 


\section{Uniform finite time convergence}

Let $R>0$. Consider perturbations $e, d: \mathbb{R}^{3} \times[0,+\infty) \rightarrow \mathbb{R}^{3}$ satisfying the regularity assumptions (7) and (10), for every $x \in \mathbb{R}^{3}$. Let $x_{0} \in \mathbb{R}^{3}$ such that $\left|x_{0}\right|<R$, let $s_{0} \in\{1,2\}$, and $\left(x, s_{d}\right)$ be the corresponding solution of (8), in closed-loop with (37) and (38), starting from $\left(x_{0}, s_{0}\right)$, and defined on $[0,+\infty)$.

As a consequence of Lemmas 3,4 and 8 , we have, for every $t \geq 0$,

$$
|x(t)| \leq \max \left(\delta_{l}(R), \delta_{l}\left(\delta_{g}(R)\right), \delta_{l}\left(\delta_{g}\left(\delta_{l}(R)\right)\right)\right),
$$

i.e.

$$
|x(t)| \leq \delta_{l}\left(\delta_{g}\left(\delta_{l}(R)\right)\right) .
$$

Thus (11) holds.

Consider now $\tau=\tau_{l}\left(\delta_{l}\left(\delta_{g}\left(\delta_{l}(R)\right)\right)\right)$, where $\tau_{l}$ is the time defined in Lemma 3. From Lemmas 3, 4 and 8 , we get, for every $t \geq \tau$,

$$
x(t)=0
$$

\section{Minimal time}

Let $x_{0} \in K$. The solution of system (8), in closed-loop with (37) and (38), starting from $x_{0}$, switches at most two times and, using (46), (47), and Lemma 8 , enters $\Gamma_{1}$ (let say, at a point $x_{1}$ ) in time less than $2 \varepsilon$. After the last switch, Lemma 3 applies, and thus, the solution reaches the origin at a time less than $T\left(x_{1}\right)+\varepsilon$. Since the gradient of $T$ is uniformly bounded (see Lemma 1), then, up to reducing $r_{0}$, we get $T\left(x_{0}\right) \leq T\left(x_{1}\right)+\varepsilon$. As 
a conclusion, the solution reaches the origin in time less than $T\left(x_{0}\right)+4 \varepsilon$.

Theorem 1 is proved.

\section{References}

1. F. Ancona and A. Bressan, Flow stability of patchy vector fields and robust feedback stabilization, SIAM J. Cont. Opt. 41, 5 (2002), 1455-1476.

2. F. Ancona and A. Bressan, Patchy vector fields and asymptotic stabilization, ESAIM Cont. Optim. Calc. Var. 4 (1999), 445-471.

3. A. Astolfi, Discontinuous control of the Brockett integrator, European J. Control 4 (1998), 49-53.

4. R. Beals, B. Gaveau and P.C. Greiner, Hamilton-Jacobi theory and the heat kernel on Heisenberg groups, J. Math. Pures Appl. 79, 7 (2000), 633-689.

5. A. Bellaïche, Tangent space in sub-Riemannian geometry, Sub-Riemannian geometry, Birkhäuser (1996).

6. A. Bensoussan and J.L. Menaldi, Hybrid control and dynamic programming, Dyn. Cont. Discrete Impulsive Syst. 3, 4 (1997), 395-442.

7. M.S. Branicky, Multiple Lyapunov functions and other analysis tools for switched and hybrid systems, IEEE Trans. Automat. Control, 43 (1998), 475482.

8. A. Bressan, Singularities of stabilizing feedbacks, Rend. Sem. Mat. Univ. Pol. Torino 56, 4 (1998), 87-104.

9. R.W. Brockett, Asymptotic stability and feedback stabilization, Differential geometric control theory, R.W. Brockett, R.S. Millman and H.J. Sussmann, ed., Boston, Birkhäuser (1983), 181-191. 
10. F.H. Clarke, Yu.S. Ledyaev, L. Rifford and R.J. Stern, Feedback stabilization and Lyapunov functions, SIAM J. Cont. Opt. 39, 1 (2000), 25-48.

11. R.T. Closkey and R.M. Murray, Exponential stabilization of driftless nonlinear control systems using homogeneous feedback, IEEE Trans. Automat. Control 42, 5 (1997), 614-628.

12. J.-M. Coron, Global asymptotic stabilization for controllable systems without drift, Math. Control Signals Syst. 5 (1992), 295-312.

13. I. Kolmanovsky and N.H. McClamroch, Developments in nonholonomic control problems, IEEE Control Systems 15 (1995), 20-36.

14. Yu.S. Ledyaev and E.D. Sontag, A remark on robust stabilization of general asymptotically controllable systems, Proc. Conf. on Information Sciences and Systems, Johns Hopkins, Baltimore (1997), 246-251.

15. E. Litsyn, Y.V. Nepomnyashchikh and A. Ponosov, Stabilization of linear differential systems via hybrid feedback controls, SIAM J. Cont. Opt. 38 (2000), $1468-1480$.

16. P. Morin, J.-B. Pomet and C. Samson, Developments in time-varying feedback stabilization of nonlinear systems, IFAC Symp. on Nonlinear Control System Design, Enschede, The Netherlands, 3 (1998).

17. P. Morin and C. Samson, Practical stabilization of driftless systems on Lie groups: the transverse function approach, IEEE Trans. Automat. Control 48 , 9 (2003), 1496-1508.

18. P. Morin and C. Samson, Exponential stabilization of nonlinear driftless systems with robustness to unmodeled dynamics, ESAIM Cont. Optim. Calc. Var. 4 (1999), 1-35.

19. L.S. Pontryagin, V.G. Boltyanskij, R.V. Gamkrelidze, E.F. Mishchenko, The mathematical theory of optimal processes, Interscience Publishers, John Wiley 
\& Sons, New York (1962).

20. C. Prieur, Uniting local and global controllers with robustness to vanishing noise, Math. Control Signals Systems 14 (2001), 143-172.

21. C. Prieur, A robust globally asymptotically stabilizing feedback: the example of the Artstein's circles, Nonlinear Control in the Year 2000, Lecture Notes in Control and Information Sciences Volume 258, Springer Verlag, London (2000), 279-300.

22. C. Prieur, Asymptotic controllability and robust asymptotic stabizability, SIAM J. Cont. Opt, to appear (2005).

23. C. Prieur and A. Astolfi, Robust stabilization of chained systems via hybrid control, IEEE Trans. Automat. Control 48, 10 (2003), 1768-1772.

24. L. Rifford, The stabilization problem: AGAS and SRS feedbacks, Optimal Control, Stabilization, and Nonsmooth Analysis, Lectures Notes in Control and Information Sciences, Volume 301, Springer Verlag, Heidelberg (2004), $173-184$.

25. E.D. Sontag, Clocks and insensitivity to small measurement errors, ESAIM Cont. Opt. Calc. Var. 4 (1999), 537-557.

26. E.D. Sontag, Stability and stabilization: Discontinuities and the effect of disturbances, Nonlinear Analysis, Differential Equations, and Control, Proc. NATO Advanced Study Institute, Montreal, F.H. Clarke, R.J. Stern, ed., Kluwer (1999), 551-598.

27. H.J. Sussmann, Subanalytic sets and feedback control, J. Diff. Eq. 31, 1 (1979), 31-52.

28. L. Tavernini, Differential automata and their discrete simulators, Nonlinear Anal. 11 (1997), 665-683. 